\title{
Decreased expression of BATF2 is significantly associated with poor prognosis in oral tongue squamous cell carcinoma
}

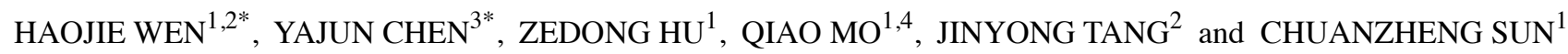 \\ ${ }^{1}$ Department of Head and Neck Surgery, The Third Affiliated Hospital of Kunming Medical University, Kunming 650118; \\ ${ }^{2}$ Department of Otorhinolaryngology - Head and Neck Surgery, The First People's Hospital of Chenzhou City, Chenzhou; \\ ${ }^{3}$ Department of Oncology, Jingzhou Hospital, Tongji Medical College, Huazhong University of Science and Technology, Jingzhou; \\ ${ }^{4}$ Department of Otorhinolaryngology - Head and Neck Surgery, The Central Hospital of Shaoyang City, Shaoyang, P.R. China
}

Received September 12, 2013; Accepted November 1, 2013

DOI: $10.3892 /$ or.2013.2863

\begin{abstract}
BATF2, also called SARI, is associated with several cancer types, and loss of BATF2 expression is frequently detected in aggressive and metastatic cancers. The expression of BATF2 was previously shown to slow the growth rate of malignant tumor cells injected into athymic nude mice, and decreased expression of BATF2 has been correlated to poor prognosis in hepatocellular carcinoma. However, the functional role of BATF2 in oral tongue squamous cell carcinoma (OTSCC) remains unknown. In the present study, we examined BATF2 expression in 16 fresh, paired OTSCC and adjacent non-tumor tissues, as well as in a normal tongue epithelial cell line and in 5 OTSCC cell lines by quantitative PCR and western blot analysis. We also evaluated BATF2 expression in 202 paraffin-embedded OTSCC and 30 adjacent non-tumor samples by immunohistochemistry, and its relationship with clinicopathological features and prognosis was investigated. We found that BATF2 expression was significantly reduced in the majority of the 16 OTSCC tumor tissues and the 5 OTSCC cell lines when compared with the non-tumor tissues and the normal tongue epithelial cell line, respectively. Consistent with these results, our immunohistochemistry analysis revealed that decreased BATF2 expression was present in 124 of the 202 cases and was significantly correlated with poor tumor differentiation $(\mathrm{P}=0.002)$. Patients with decreased BATF2 expression showed reduced survival when compared to those with high expression $(\mathrm{P}<0.001)$. Multivariate analysis revealed that BATF2 expression is an independent predictor of overall survival $(\mathrm{P}=0.001)$. These results demonstrate that $\mathrm{BATF} 2$ plays a tumor-suppressor role in the development of OTSCC
\end{abstract}

Correspondence to: Dr Chuanzheng Sun, Department of Head and Neck Surgery, The Third Affiliated Hospital of Kunming Medical University, 519 Kunzhou Road, Kunming 650118, P.R. China

E-mail: scz008@126.com

${ }^{*}$ Contributed equally

Key words: BATF2, tongue, squamous cell carcinoma, prognosis and that BATF2 may serve as a prognostic biomarker and potential therapeutic target for this disease.

\section{Introduction}

Oral tongue squamous cell carcinoma (OTSCC), the most common type of oral cancer, exhibits increased incidence and poor prognosis $(1,2)$. OTSCC is significantly more aggressive than other forms of oral cancer, as it has a propensity for rapid local invasion, metastasis and a high recurrence rate $(3,4)$. The prognosis for patients with OTSCC has not strikingly improved over the past 3 decades, even with combined treatment involving surgery, chemotherapy and radiation; the 5-year overall survival rate of OTSCC is $\sim 50-60 \%(2,5-7)$. Therefore, it is critical to develop novel biomarkers for predicting prognosis and for establishing targeted treatments for patients with OTSCC.

BATF2, also known as SARI (suppressor of AP-1, regulated by IFN) and a member of the BATF subfamily of bZIP proteins, was cloned and identified in 2008. The BATF2 gene is located at 11q12-13. Steady-state BATF2 mRNA expression was detected in multiple lineage-specific normal cells, but not in their transformed/tumorigenic counterparts (8). Furthermore, overexpression of BATF2 was found to inhibit proliferation and induce apoptosis in cancer cells but not in normal cells (8). In contrast, BATF2 downregulation was found to promote tumor proliferation and metastasis in lung adenocarcinoma (9). Recently, decreased expression of BATF2 was found to be associated with poor prognosis in hepatocellular carcinoma (HCC) (10) and colorectal carcinoma (11). Thus, it is likely that BATF2 functions as a tumor-suppressor in cancer development; however, no data regarding BATF2 expression and its correlation with OTSCC are available.

In the present study, we analyzed the expression of BATF2 in OTSCC using quantitative PCR, western blotting and immunohistochemistry and investigated the relationship between its expression and the clinicopathological features of the OTSCC patients. We also evaluated the potential prognostic value of BATF2 in the postoperative survival of OTSCC patients. Our data showed that BATF2 plays an important role in the development of OTSCC and may be considered as a candidate tumor-suppressor and a prognostic marker for patients with OTSCC. 


\section{Materials and methods}

Patients and clinical tissue specimens. The present study was approved by the Ethics Committee of The Third Affiliated Hospital of Kunming Medical University, and informed consent was obtained from all participants prior to enrollment. A total of 16 paired OTSCC tissues and adjacent non-tumor tissues (distance from the tumor of $>2 \mathrm{~cm}$ ) were collected from OTSCC patients who had undergone surgical resection at The Third Affiliated Hospital of Kunming Medical University between May 2010 and August 2010. The fresh tissues were immediately immersed in RNAlater (Ambion, Inc., Austin, TX, USA) after surgical resection, stored at $4^{\circ} \mathrm{C}$ overnight to allow thorough penetration of the tissue and then frozen at $-80^{\circ} \mathrm{C}$ until RNA and protein extraction was performed. An additional 202 paraffin-embedded OTSCC samples, which were pathologically and clinically diagnosed between January 2000 and December 2005 at The Third Affiliated Hospital of Kunming Medical University, were collected for immunohistochemistry. These samples were from 112 males and 90 females, with a median age of 53 years (ranging from 21 to 78 years), and none of these patients received radiotherapy or chemotherapy prior to surgery. The clinicopathological features and BATF2 expression levels for the 202 patients are shown in Table I. The histological differentiation of the samples was determined according to the criteria of the World Health Organization. The tumor $(\mathrm{T})$ classification, node $(\mathrm{N})$ classification and clinical tumor-node-metastasis (TNM) stage were assessed according to the TNM classification of the American Joint Committee on Cancer (AJCC) (12).

Cell lines and cell culture. A normal tongue epithelial cell line (NTEC1) was established by culturing normal tongue squamous epithelium from a non-tumor patient in keratinocyte/serumfree medium (Invitrogen Life Technologies, Carlsbad, CA, USA). OTSCC cell lines were purchased from the American Type Culture Collection (ATCC, Manassas, VA, USA) (CAL27, SCC-25 and SCC-9 cells) or from the Committee of the Type Culture Collection of the Chinese Academy of Sciences (Shanghai, China) (TCA8113 and TSCCA cells). All cells were cultured in DMEM/F12 (Invitrogen Life Technologies) supplemented with $10 \%$ fetal bovine serum (HyClone, Logan, UT, USA), penicillin $(100 \mathrm{U} / \mathrm{ml})$ and streptomycin $(100 \mathrm{U} / \mathrm{ml})$ at $37^{\circ} \mathrm{C}$ in a humidified $5 \% \mathrm{CO}_{2}$ incubator.

Quantitative ( $q$ )PCR. Total RNA from the cell lines and human tissues was extracted using TRIzol reagent (Invitrogen Life Technologies). After reverse transcription of the total RNA, the first-strand cDNA was used as a template for detecting the expression of BATF2. qPCR was performed with an ABI PRISM 7900HT sequence detection system. The housekeeping gene GAPDH was used as an internal control to normalize the expression levels of BATF2. The primer sequences were: 5'-AGACCCCAAGGAGCAACA-3' (sense), and 5'-CTTTTTCCAGAGACTCGTGCT-3' (antisense) for BATF2, and 5'-CTCCTCCTGTTCGACAGTCAGC-3' (sense), and 5'-CCCAATACGACCAAATCCGTT-3' antisense for GAPDH. To ensure the reproducibility of the results, all experiments were repeated 3 times. The data were analyzed using the comparative threshold cycle $\left(2^{-\Delta \Delta \mathrm{CT}}\right)$ method.
Table I. Correlation between the expression of BATF2 and the clinicopathological variables of the 202 patients with OTSCC.

\begin{tabular}{lrrr}
\hline \multirow{2}{*}{$\begin{array}{l}\text { Clinicopathological } \\
\text { variables }\end{array}$} & \multicolumn{2}{l}{ BATF2 expression n (\%) } & \\
\cline { 2 - 3 } Gender & No/low & High & P-value \\
Male & & & 0.943 \\
Female & $69(61.6)$ & $43(38.4)$ & \\
Age (years) & $55(61.1)$ & $35(38.9)$ & \\
$\quad \leq 50$ & & & 0.513 \\
$>50$ & $53(57.8)$ & $37(42.1)$ & \\
Tobacco history & $71(63.5)$ & $41(36.5)$ & \\
Smoker & & & 0.429 \\
Nonsmoker & $44(57.3)$ & $32(42.7)$ & \\
Clinical TNM stage & $80(63.5)$ & $46(36.5)$ & \\
I & & & 0.198 \\
II & $34(52.3)$ & $31(47.7)$ & \\
III & $52(65.8)$ & $27(34.2)$ & \\
IV & $27(61.4)$ & $17(38.6)$ & \\
T classification & $11(78.6)$ & $3(21.4)$ & \\
T1-2 & & & 0.162 \\
T3-4 & $108(59.7)$ & $73(40.3)$ & \\
N classification & $16(76.2)$ & $5(23.8)$ & \\
N0 & & & 0.866 \\
N+ & $93(60.8)$ & $60(39.2)$ & \\
Histological & $31(63.3)$ & $18(36.7)$ & \\
differentiation & & & 0.002 \\
Well & & & \\
Moderate & $79(54.1)$ & $67(45.9)$ & \\
Poor & $37(92.5)$ & $10(7.5)$ & \\
\hline OTSCC, & $8(88.9)$ & $1(11.1)$ & \\
\hline
\end{tabular}

OTSCC, oral tongue squamous cell carcinoma; $\mathrm{T}$, tumor; $\mathrm{N}$, node; TNM, tumor-node-metastasis.

Western blot analysis. Western blot analysis was performed as previously described (10). When relevant, the blots were probed with an anti-BATF2 mouse monoclonal antibody (1:1,000 dilution; Abnova Corporation, Taiwan) and an anti-GAPDH mouse monoclonal antibody (1:1,000 dilution; Santa Cruz Biotechnology, Inc., Santa Cruz, CA, USA). The signals were detected using enhanced chemiluminescence (ECL) (Amersham Pharmacia Biotech, Piscataway, NJ, USA) according to the manufacturer's suggested protocol.

Immunohistochemical staining. In brief, paraffin-embedded tongue tissue specimens were cut into $4-\mu \mathrm{m}$ sections and incubated at $65^{\circ} \mathrm{C}$ for $1 \mathrm{~h}$. All sections were deparaffinized with xylene and rehydrated in a graded ethanol series. After treatment with $3 \% \mathrm{H}_{2} \mathrm{O}_{2}$ for 15 min to block the endogenous peroxidase, the sections were submerged in EDTA antigen retrieval buffer ( $\mathrm{pH} \mathrm{8.0)}$ and microwaved. Then, the sections were treated with normal goat serum for $30 \mathrm{~min}$ to reduce any nonspecific binding and were incubated with an anti-BATF2 


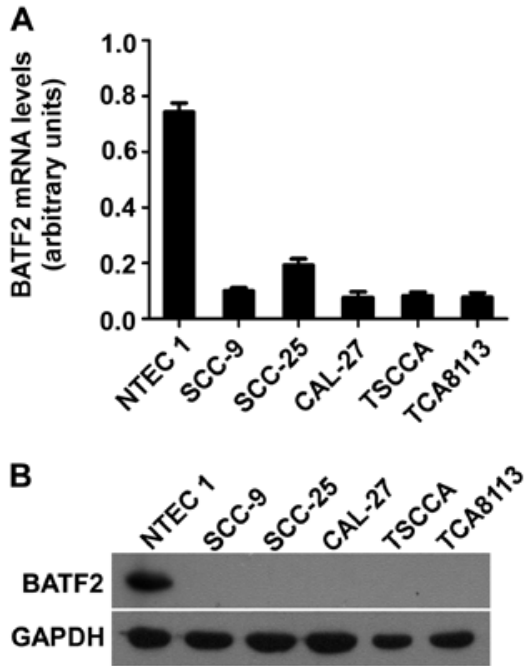

Figure 1. BATF2 expression is downregulated in OTSCC cell lines. (A) mRNA from 5 OTSCC cell lines (SCC-9, SCC-25, CAL-27, TSCCA and TCA8113) and a normal tongue epithelial cell line (NTEC1) was extracted and assayed by qPCR. The expression levels were normalized to that of GAPDH. The average of 3 independent experiments in triplicate is shown in the graph; error bars indicate $\pm \mathrm{SD}$. (B) The protein expression of BATF2 in the 5 OTSCC cell lines and NTEC1 was also examined by western blot analysis. GAPDH was used to ensure equal protein loading. OTSCC, oral tongue squamous cell carcinoma.

mouse monoclonal antibody (1:1,000; Abnova Corporation) overnight at $4^{\circ} \mathrm{C}$. After washing, the sections were incubated with a biotinylated anti-rabbit secondary antibody, followed by further incubation with streptavidin-horseradish peroxidase (both from Dako) at $37^{\circ} \mathrm{C}$ for $30 \mathrm{~min}$. To produce a color reaction, diaminobenzidine (DAB) was used. For the negative controls, the antibody was replaced with normal goat serum. The immunostained samples were evaluated by two independent pathologists who did not have knowledge of the clinical data. According to previous studies $(10,13)$, the intensity of the cells expressing BATF2 was scored as follows: 0 (no staining), 1 (weak staining, light yellow), 2 (moderate staining, yellow-brown) or 3 (strong staining, brown). The percentage of positive staining was scored as follows: 0 (no expression), $1(1-25 \%), 2(26-50 \%), 3(51-75 \%)$ or $4(>75 \%)$. The BATF2 expression level was calculated as the intensity score plus the proportion score and was divided into 4 grades: - (score of 0 and 1$),+($ score of 2 and 3$),++$ (score of 4 and 5) and +++ (score of 6 and 7). In the present study, '-' and ' + ' represented no/low expression (score of 0-3), whereas ' ++ ' and ' +++ ' were regarded as high expression (score of 4-7).

Statistical analysis. Overall survival was calculated from the date of surgery until the day of death or the last date in the medical records on which the patient was reported to be alive. Disease-free survival was calculated from the date of surgery until the last follow-up date on which the patient was not found to have tumor recurrence. A paired samples t-test was used to compare the mRNA and protein expression of BATF2 in the paired OTSCC and adjacent non-tumor tissue samples. A $\chi^{2}$ test was used to analyze the relationship between BATF2 expression and the clinicopathological features of the OTSCC patients. Survival curves were plotted using the
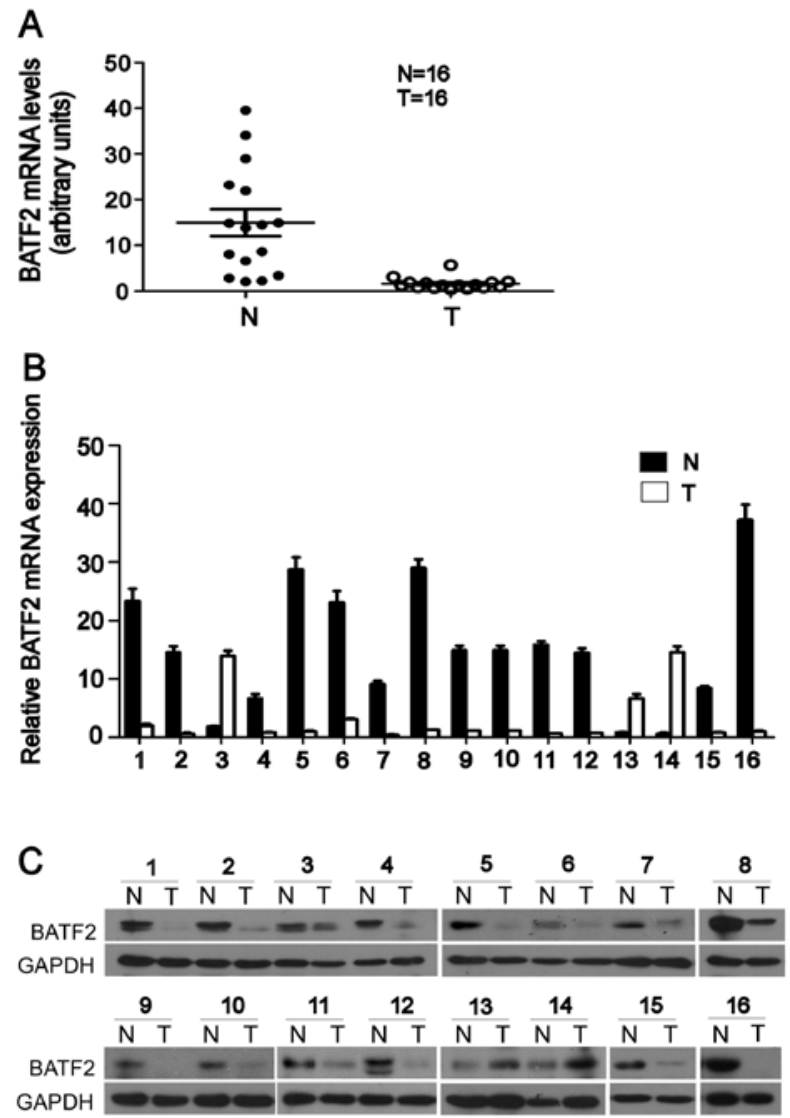

Figure 2. BATF2 expression is downregulated in fresh OTSCC tissues (A and B) The relative mRNA expression levels of BATF2 (BATF2/GAPDH) in 16 paired OTSCC and adjacent non-tumor tissues were determined by qPCR. Horizontal lines represent the mean. (C) The protein expression levels of BATF2 in the 16 paired OTSCC and matched adjacent non-tumor tissues were also examined by western blot analysis. GAPDH was used as an internal control. T, OTSCC tissue; $\mathrm{N}$, matched adjacent non-tumor tissue. OTSCC, oral tongue squamous cell carcinoma.

Kaplan-Meier method and compared with the log-rank test. The Cox proportional hazard regression model was used for univariate and multivariate analyses to explore the influence of the clinicopathological variables and BATF2 expression on survival. SPSS 18.0 software (SPSS, Chicago, IL, USA) was used for all statistical analyses, and a P-value of $<0.05$ was considered to indicate a significant result.

\section{Results}

BATF2 expression is decreased in the OTSCC cell lines. To examine the expression levels of BATF2, qPCR and western blot analysis were conducted using the normal tongue epithelial cell line NTEC1 and the OTSCC cell lines SCC-9, SCC-25, CAL-27, TSCCA and TCA8113. qPCR revealed lower expression of BATF2 mRNA in all of the cancer cell lines when compared to the NTEC1 cell line (Fig. 1A). As shown in Fig. 1B, high expression of the BATF2 protein was observed in the NTEC1 cell line, whereas the expression of the BATF2 protein was significantly lower, i.e., at an undetectable level, in the OTSCC cell lines. These findings are consistent with those from our qPCR experiment. Thus, our data indicated that BATF2 was downregulated at both the mRNA and protein levels in the OTSCC cell lines. 

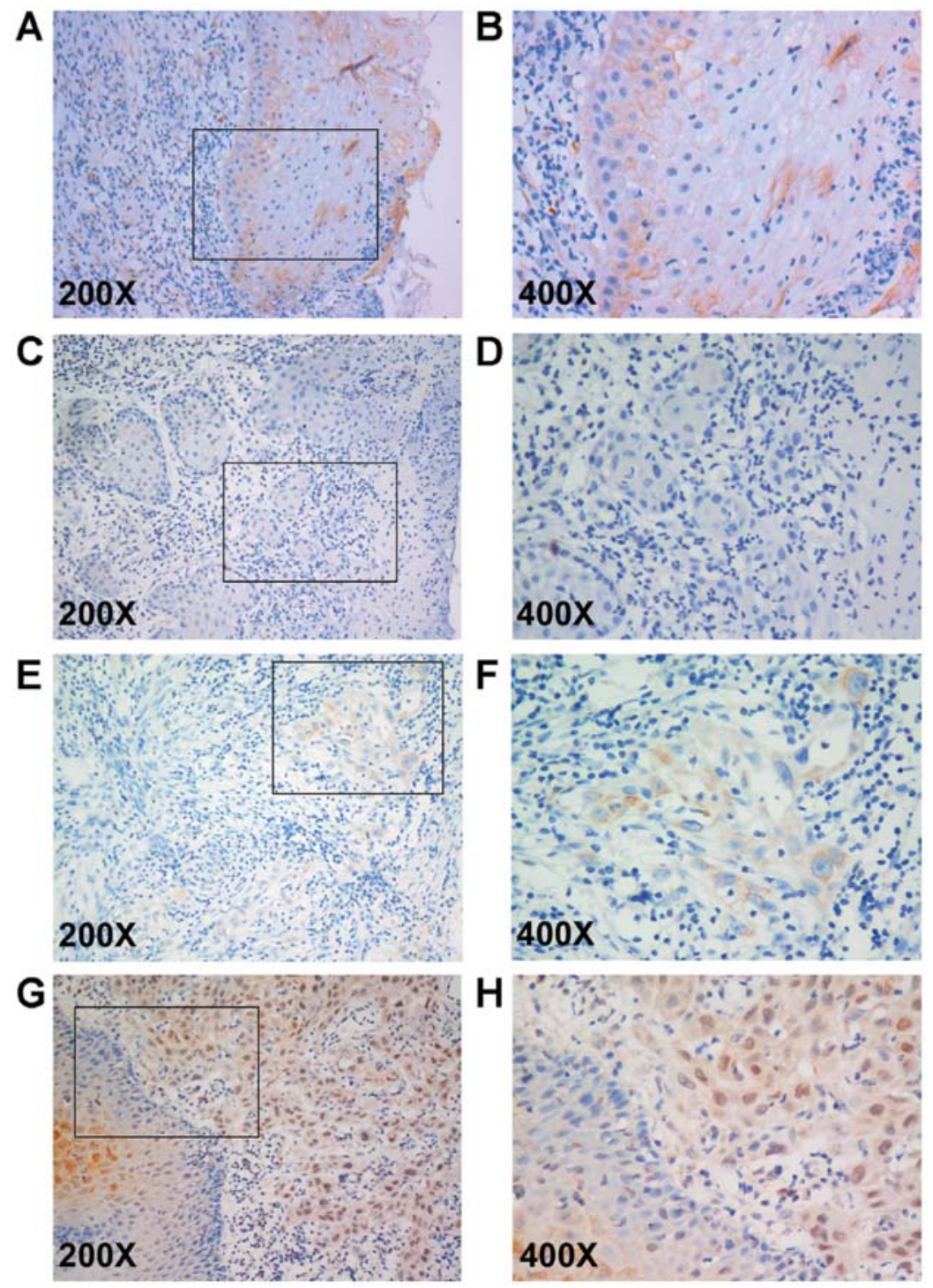

Figure 3. BATF2 expression is decreased in paraffin-embedded OTSCC tissues. Paraffin-embedded OTSCC samples were subjected to immunohistochemical staining with an antibody against BATF2. (A and B) High expression of the BATF2 protein in adjacent non-tumor tissues. (C and D) Negative expression of BATF2 in OTSCC tissues. (E and F) Low expression of BATF2 in poorly differentiated OTSCC tissues (+). (G and H) High expression of BATF2 in welldifferentiated OTSCC tissues (+++). Original magnification, (A, C, E and G x200) and (B, D, F and H x400). OTSCC, oral tongue squamous cell carcinoma.

BATF2 expression is downregulated in fresh OTSCC tissues. To determine the expression of BATF2 in OTSCC tissues, qPCR and western blot analysis were performed using 16 OTSCC tissues and their matched adjacent non-tumor tissues. BATF2 mRNA was found to be significantly downregulated in $13(81.25 \%, 13 / 16)$ of the OTSCC tissues when compared with their matched adjacent non-tumor tissues $(\mathrm{P}=0.001)$ (Fig. 2A and B). Similarly, the BATF2 protein was also significantly downregulated in $14(87.5 \%, 14 / 16)$ of the tumor samples when compared with the adjacent non-tumor tissues from the same patients $(\mathrm{P}<0.001)$ (Fig. 2C).

Correlation between BATF 2 expression and clinicopathological features. To verify the relationship between BATF2 and the development of OTSCC, we examined BATF2 expression in 202 paraffin-embedded OTSCC samples and 30 adjacent non-tumor tissue specimens by immunohistochemistry. As a result, among the 30 non-tumor tissue specimens, 18 cases
(60\%) showed high expression (Fig. 3A and B), 8 cases $(26.7 \%)$ showed low expression and 4 cases $(13.3 \%)$ showed negative expression. However, among the 202 OTSCC samples, 20 cases $(9.9 \%)$ showed negative expression (Fig. 3C and D), 104 cases $(51.5 \%)$ showed low expression (Fig. 3E and F) and $78(38.6 \%)$ cases showed high expression (Fig. 3G and H). As shown in Table I, the BATF2 expression level was significantly correlated with histological differentiation $(\mathrm{P}=0.002)$ but not with age, gender, tobacco history, $\mathrm{T}$ classification, $\mathrm{N}$ classification or clinical TNM stage.

BATF2 expression and prognosis. The patients with no/low BATF2 expression had a significantly poorer 5-year overall survival rate and disease-free survival rate than those with high BATF2 expression (50.5 vs. 89.7\%, P<0.001, Fig. 4A) (42.8 vs. $86.5 \%, \mathrm{P}<0.001$, Fig. $4 \mathrm{~B}$ ). Univariate Cox regression analyses demonstrated that clinical TNM stage, histological differentiation and BATF2 expression level were significantly 
A
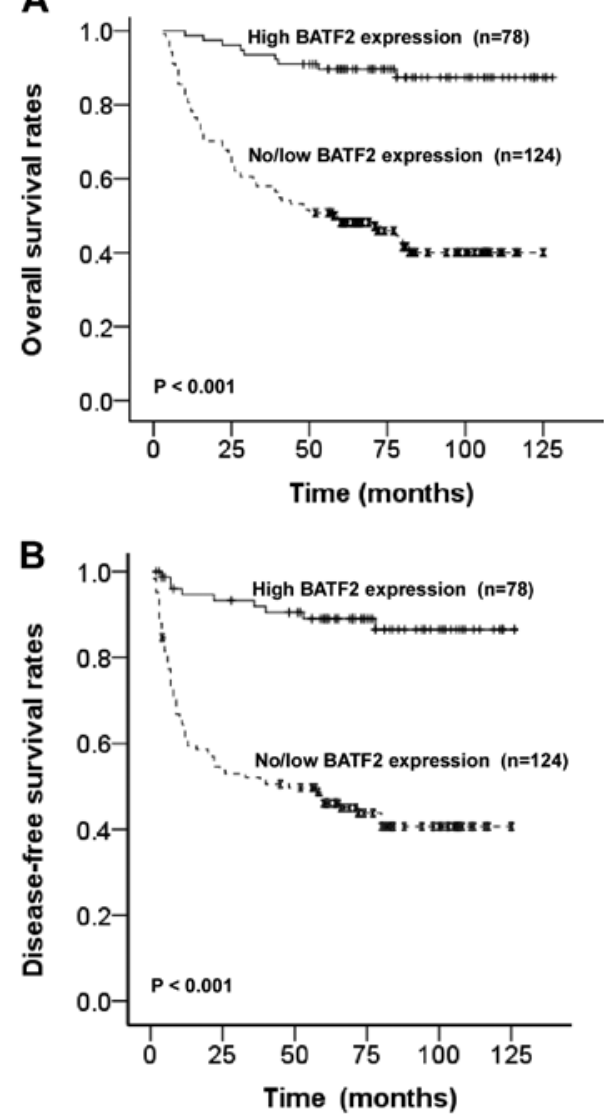

Figure 4. BATF2 expression is positively associated with the overall survival and disease-free survival rates. Kaplan-Meier plots show the (A) overall survival rate and (B) disease-free survival rate of the 202 patients with OTSCC according to the level of BATF2 expression in their tumor tissues. Patients were grouped according to the BATF2 expression level. OTSCC, oral tongue squamous cell carcinoma.

associated with overall survival. Furthermore, multivariate Cox regression analyses revealed that the above mentioned 3 factors are independent predictors for the overall survival of OTSCC patients $(\mathrm{P}=0.000,0.001$ and 0.001 , respectively; Table II). These findings indicate that a high level of BATF2 expression is a biomarker for better prognosis in patients with OTSCC.

\section{Discussion}

Despite advances in the early diagnosis and management of cancer, the outcome for patients with OTSCC has not significantly improved over the last 3 decades $(2,6,7)$. Therefore, it is critical to identify biomarkers and molecular targets for these patients. Basic leucine zipper transcription factor ATF-like (BATF), BATF2 and BATF3 belong to the activator protein 1 (AP-1) family of transcription factors, which regulate numerous cellular processes (14). Su et al (8) detected steady-state expression of BATF 2 mRNA in many normal cell lines, such as melanocytes, astrocytes, breast and prostate epithelial cells and pancreatic mesothelial cells, but not in their malignant counterparts. The overexpression of BATF2 was shown to induce profound growth inhibition and apoptosis in malignant glioma, melanoma and prostate cancer cell lines, with no effect on the survival of the corresponding normal cells. The
Table II. Univariate and multivariate analyses using the Cox regression model.

\begin{tabular}{lcc}
\hline Variables & Hazard risk $(95 \% \mathrm{CI})$ & P-value \\
\hline Univariate & & \\
Gender & $1.167(0.856-1.589)$ & 0.329 \\
Age & $1.231(0.907-1.670)$ & 0.182 \\
T classification & $1.151(0.918-1.442)$ & 0.223 \\
N classification & $0.981(0.655-1.471)$ & 0.928 \\
Clinical TNM stage & $1.423(1.095-1.848)$ & 0.008 \\
Histological differentiation & $1.459(1.186-1.793)$ & 0.020 \\
Expression of BATF2 & $1.507(1.155-1.966)$ & 0.002 \\
Multivariate & & \\
Clinical TNM stage & $1.541(1.363-1.743)$ & 0.000 \\
Histological differentiation & $1.424(1.158-1.750)$ & 0.001 \\
Expression of BATF2 & $1.564(1.210-2.022)$ & 0.001
\end{tabular}

CI, confidence interval; $\mathrm{T}$, tumor; $\mathrm{N}$, node; TNM, tumornode-metastasis.

growth inhibitory effect of BATF2 was further confirmed by its ability to slow the growth rate of DU-145 prostate cancer cells injected into athymic nude mice.

However, BATF2 expression in OTSCC has not been reported. In the present study, the mRNA and protein expression of BATF2 was significantly lower in the OTSCC cell lines and tissues than in normal tongue epithelial cells or adjacent non-tumor tissues (Figs. 1 and 2). In agreement with these results from our qPCR and western blot analysis, little to no BATF2 expression was observed by immunohistochemistry in $61.4 \%$ of the OTSCC samples (124/202). Furthermore, decreased BATF2 expression was significantly associated with poor histological differentiation in the OTSCC tissues (Table I, Fig. 3). In contrast to the research of Ma et al (10), we did not find that age or tumor size of the patients with OTSCC was significantly correlated with the level of BATF2 expression, indicating that decreased BATF2 expression may exert different effects on the development of different neoplasms. Survival analysis showed that no or low BATF2 expression was significantly correlated with poor overall survival and disease-free survival $(\mathrm{P}<0.001)$. Multivariate Cox regression analysis further confirmed that the BATF2 expression level is an independent prognostic factor, with a hazard risk of 1.564 (95\% CI, 1.210-2.022). All of these results support the hypothesis that BATF2 functions as a tumor-suppressor gene in the development of OTSCC.

Nevertheless, the mechanism by which BATF2 expression is downregulated in solid tumors and the relationship between its decreased expression and poor prognosis are not fully understood. Mutation of tumor-suppressor genes, particularly in exons, is one of the most notable features that may lead to gene inactivation. In the study performed by Ma et al (10), no mutation was detected in any of the 3 exons of the BATF2 gene in 5 HCC cell lines and 8 HCC tumor tissues. Wang et al (9) reported that loss of BATF2 expression initiates the epithelial-mesenchymal transition, which is visualized by 
repression of E-cadherin and upregulation of vimentin in lung adenocarcinoma cell lines and clinical lung adenocarcinoma specimens. Using a human lung xenograft mouse model, the knockdown of endogenous BATF2 in human carcinoma cells was found to lead to the development of multiple lymph node metastases by modulating the (GSK)-3 $\beta-\beta$-catenin signaling pathway. Huang et al (15) demonstrated that a BCR-ABL kinase inhibitor or siRNA specific to BCR-ABL upregulated BATF2 mRNA expression in human leukemia cells. Both JAK/STAT and RAS/MAPK signaling inhibitors (AG490 and PD98059, respectively) upregulated BATF2 mRNA expression, while the PI3K/AKT pathway inhibitor LY294002 had no such effect. Therefore, this group reported that BATF2 mRNA expression was suppressed by BCR-ABL through the downstream RAS/MAPK and JAK/STAT signaling pathways in human leukemia cells. Dash et al (16) found that BATF2 selectively suppressed the transcription of CCN1, a secretory integrin-binding protein that regulates angiogenesis, cell adhesion, migration, proliferation, survival and apoptosis. Hence, BATF2 inhibited CCN1-induced anchorage-independent growth and invasion in breast cancer, malignant glioma and metastatic melanoma cells by inhibiting the activation of MAP kinase and the PI3/AKT kinases. The AP-1 transcription factor BATF3 is required for the homeostatic development of classical CD $8 \alpha^{+}$dendritic cells, which prime CD8 T-cell responses against intracellular pathogens. Recently, Tussiwand et al (17) identified an alternative pathway that results from the molecular compensation of BATF3 by BATF2, which is induced by cytokines in response to infection. Whether this pathway is related to carcinogenesis remains elusive.

In summary, BATF2 expression was decreased in most of the cases of OTSCC analyzed in the present study, and lower BATF2 expression was found to be related to poor histological differentiation and poor prognosis in patients with OTSCC, demonstrating that BATF2 may serve as a tumor-suppressor gene in the development of OTSCC and that BATF2 may be used as a prognostic biomarker and therapeutic target for OTSCC. However, the exact mechanism by which the expression of BATF2 is decreased and how BATF2 functions in the development of OTSCC remain to be explored.

\section{Acknowledgements}

The present study was partly supported by grants from the National Natural Science Foundation of China (nos. 30960444 and 81260402), and the Special Foundation of High Levels of Health Technical Personnel Training in Yunnan Province (no. D-201243).

\section{References}

1. Jemal A, Siegel R, Xu J and Ward E: Cancer statistics, 2010. CA Cancer J Clin 60: 277-300, 2010.

2. Shiboski CH, Schmidt BL and Jordan RC: Tongue and tonsil carcinoma: increasing trends in the U.S. population ages 20-44 years. Cancer 103: 1843-1849, 2005.

3. Franceschi D, Gupta R, Spiro RH and Shah JP: Improved survival in the treatment of squamous carcinoma of the oral tongue. Am J Surg 166: 360-365, 1993.

4. Yuen AP, Lam KY, Chan AC, et al: Clinicopathological analysis of elective neck dissection for N0 neck of early oral tongue carcinoma. Am J Surg 177: 90-92, 1999.

5. Myers JN, Elkins T, Roberts D and Byers RM: Squamous cell carcinoma of the tongue in young adults: increasing incidence and factors that predict treatment outcomes. Otolaryngol Head Neck Surg 122: 44-51, 2000.

6. Wen Y, Dai X, Wang C, et al: A retrospective clinical study of 6539 cases of malignant oral-maxillofacial tumors. Hua Xi Kou Qiang Yi Xue Za Zhi 19: 296-299, 2001 (In Chinese).

7. Goldstein DP, Bachar GY, Lea J, et al: Outcomes of squamous cell cancer of the oral tongue managed at the Princess Margaret Hospital. Head Neck 35: 632-641, 2013.

8. Su ZZ, Lee SG, Emdad L, et al: Cloning and characterization of SARI (suppressor of AP-1, regulated by IFN). Proc Natl Acad Sci USA 105: 20906-20911, 2008.

9. Wang C, Su Y,Zhang L, et al: The function of SARI in modulating epithelial-mesenchymal transition and lung adenocarcinoma metastasis. PLoS One 7: e38046, 2012.

10. Ma H, Liang X, Chen Y, et al: Decreased expression of BATF2 is associated with a poor prognosis in hepatocellular carcinoma. Int J Cancer 128: 771-777, 2011.

11. Liu ZB, Yang Y, Ye XG, Wang L, Tian PY and Zhang YY: Expression and significance of SARI and CCN1 in human colorectal carcinomas. Zhonghua Yi Xue Za Zhi 91: 2397-2401, 2011 (In Chinese).

12. Edge SB, Byrd DR, Compton CC, Fritz AG, Greene FL and Trotti A (eds): AJCC Cancer Staging Manual. 7th edition. Springer, New York, 2010.

13. Soumaoro LT, Uetake H, Higuchi T, Takagi Y, Enomoto M and Sugihara K: Cyclooxygenase-2 expression: a significant prognostic indicator for patients with colorectal cancer. Clin Cancer Res 10: 8465-8471, 2004.

14. Murphy TL, Tussiwand R and Murphy KM: Specificity through cooperation: BATF-IRF interactions control immune-regulatory networks. Nat Rev Immunol 13: 499-509, 2013.

15. Huang Q, Yang Y, Li X and Huang S: Transcription suppression of SARI (suppressor of AP-1, regulated by IFN) by BCR-ABL in human leukemia cells. Tumour Biol 32: 1191-1197, 2011.

16. Dash R, Su ZZ, Lee SG, et al: Inhibition of AP-1 by SARI negatively regulates transformation progression mediated by CCN1. Oncogene 29: 4412-4423, 2010.

17. Tussiwand R, Lee WL, Murphy TL, et al: Compensatory dendritic cell development mediated by BATF-IRF interactions. Nature 490: 502-507, 2012. 